\title{
Class of initial letter as a cue to correctness in verbal discrimination
}

\author{
ROBERT L. GREEN and MARIAN SCHWARTZ \\ University of Wisconsin, Madison, Wisconsin 59706
}

\begin{abstract}
In a verbal discrimination task, each pair consisted of a word beginning with a vowel (A, E, 0 , or $U$ ) paired with a word beginning with a consonant (C, F, G, or S). When all the initial-vowel words were correct, performance was significantly better than when correctness was randomly determined. When all the initial-consonant words were correct, performance was not significantly different from the random control. Awareness of (i.e., ability to verbalize) the initial-vowel rule was not necessary for its effective utilization during VD learning, but only those few subjects who could verbalize the rule showed evidence of generalizing it to new words.
\end{abstract}

The effect of rules upon learning in the verbal discrimination (VD) paradigm has been investigated by Kausler and his associates (Deichmann, Minnigerode. \& Kausler, 1970; Kausler, Erber, \& Olson, 1970; Kausler \& Farzanegan, 1969; Kausler, Majcher, \& Conover, 1975) and by Schwartz (1974). In all studies, a list of pairs was presented and the subjects' task was to learn which member of each pair was correct. In the various experimental conditions, correctness was governed by a list-wide rule; in a control condition, no rule applied and learning could occur only by rote. It was found that learning of word pairs was facilitated when correctness was governed by rules based on pre-experimental frequency (Deichmann et al., 1970; Kausler \& Farzanegan, 1969), length of word (Deichmann et al., 1970), taxonomic category (Kausler, Erber, \& Olson, 1970), and relative position of the pair members along the evaluative dimension of connotative meaning (Schwartz, 1974). Also, the learning of pairs of dot patterns was facilitated by the presence of common features among the correct items (Kausler, Majcher, \& Conover, 1975).

The present study was designed to investigate further the effect of a list-wide rule upon VD learning. For all subjects, one member of each word pair in the list started with a vowel (A. E, O, or U), and the other member started with a consonant (C, F, G, or S). In the rule group, class of initial letter determined correctness. The rule group was divided into two subgroups: vowel-correct (i.e., initial-vowel words were correct) and consonant-correct. In the control group. half the correct words started with each class of initial letter, and no rule applied. The VD task was followed by a post-test designed to investigate possible generalization of the rule to new words. Finally, subjects were questioned in an effort to determine whether they had become aware of the rule.

Robert L. Green is now at the Community Service Society. New York. N. Y.

\section{METHOD}

\section{Materials}

A VD list of 24 pairs was constructed from 48 five-letter nouns. Six nouns began with each of the Letters A, E, O, U, C. F, G, and $S$. In selecting the words, an attempt was made to minimize formal similarity in other than the first-letter position, and also to minimize meaningful similarity. Two initial-vowel words and two initial-consonant words were of one syllable; the rest were of two syllables. The six words starting with a given vowel were paired with words starting with all four of the consonants, and vice versa. The two members of each pair were approximately matched for pre-experimental frequency and for imagery value. There were four different orders of presentation of the 24 pairs. The orders were random except for certain restrictions; e.g., that no more than two consecutive pairs could contain words with the same initial letter.

Four lists were constructed-one each for the vowel-correct and consonant-correct conditions. and two for the control condition-which differed only in which member of each pair was designated correct. In Control List 1 , correctness was randomly determined except for certain restrictions such as that half the words starting with each initial letter were correct. Whichever pair member was correct in Control List 1 was incorrect in Control List 2. All lists were constructed so that the alphabetically prior member of the pair was correct in half the pairs, and also the top member was correct in half the pairs.

The post-test list used 16 eight-letter adjectives of three or four syllables, arranged to form eight pairs in which one word began with a vowel and the other with a consonant. Two post-test words started with each of the vowels $A, E, O$, and $U$; one post-test word started with each of the consonants $C, F, G$, and $S$; the remaining four words each started with a new initial consonant (H. M. R. and $\mathrm{T})$. With respect to consonants, therefore, four of the post-test pairs tested generalization to new words starting with old consonants. and the other four tested generalization to new members of the consonant class. Four versions of the post-test, differing with respect to pairings and orders, were constructed.

\section{Procedure and Subjects}

Subjects were run individually. All received the same standard VD instructions. which made no mention of rules, of initial letters. or of the later post-test. The anticipation procedure was used to present the VD list for four trials on a Stowe memory drum at a $3: 1$-sec rate. with a 4 -sec intertrial interval. In the study interval. the two members of the pair were shown in top-bottom position; in the feedback interval the correct word was shown to the right of the pair. Lowercase pica type was used.

After the last VD trial, the subject was informed that eight new pairs of words would be shown only once, and was instructed to call out one member of each pair. "whichever one you guess would have been correct if these pairs had been in the list you just saw. " The eight post-test pairs were then presented at a 5 -sec rate. without 
feedback. After the post-test, the subjects in the rule group were asked first "Do you have any comments about any aspect of this experiment?" and then "While you were learning the original list, did it seem to you that the correct words had something in common?"

The subjects were 48 students from an introductory psychology course. Using a block-randomization procedure, 12 were assigned to each of the four subgroups (vowel-correct, consonant-correct, Control List 1, and Control List 2). Within each subgroup, equal numbers of subjects were assigned to each of the four starting orders of the VD list and to each of the four versions of the post-test.

\section{RESULTS}

On the four VD trials combined, the mean number of correct responses was 71.04 for the rule group $(\mathrm{SD}=8.14)$, and 62.75 for the control group $(\mathrm{SD}=8.36)$. Analysis of variance indicated that the difference was significant, $F(1,46)=12.11, p<.01$. Within the rule group, mean correct was 74.58 for the vowel-correct subgroup $(\mathrm{SD}=6.30)$ and 67.50 for the consonant-correct subgroup $(S D=8.46)$, a significant difference, $F(1,22)=5.41, p<.05$. For the purpose of comparing each rule subgroup with the control condition, the control-group lists were divided into two subsets-the 12 pairs in which the initial-vowel word was correct and the 12 pairs in which the initial-consonant word was correct-and the number of correct responses for each subset was multiplied by 2 . The vowel-correct list was then compared with the vowel-correct subset of the control list (mean correct $=63.75$ ), yielding a significant difference, $F(1,34)=8.81, p<.01$. A corresponding analysis compared the consonant-correct list with the consonant-correct subset of the control list (mean correct $=61.75$ ); here, the difference failed to reach conventional significance levels, $F(1,34)=3.07$, $.05<\mathrm{p}<.10$.

The subjects' answers to the two final questions were used to assess awareness of the initial-letter rule during VD learning. Of the 12 vowel-correct subjects, four stated that the correct words started with vowels, and those four were accordingly labeled "Aware"; of the 12 consonant-correct subjects, none stated that the correct words started with consonants. In order to determine whether the four aware subjects accounted for the significant superiority of the vowel-correct over the control group on the VD list, an additional analysis was performed excluding those four subjects. Mean correct responses for the remaining eight vowel-correct subjects, none of whom had stated correct or partly correct rules, was 73.38. This was significantly higher than the mean for the vowel-correct subset of the control list, $F(1,30)=$ 4.74, $\mathrm{p}<.05$.

The post-test and final-inquiry results were consistent: i.e., all of the four aware subjects and none of the other 20 rule-group subjects conformed to the VD initial-letter rule on all eight post-test responses. With data for the four aware subjects deleted, the remaining vowel-correct, consonant- correct, and control subjects were compared as to their preference for initial-vowel vs. initial-consonant words on the post-test. Neither on the old-consonant nor on the new-consonant items did any significant difference emerge.

\section{DISCUSSION}

When the correct member of each pair started with a vowel and the incorrect member with a consonant, VD learning was signiticantly above the level of the control condition. When the list incorporated the reverse rule. VD learning was not significantly facilitated. This outcome can most reasonably be interpreted as the net effect of the following two asymmetries: (a) rules applied to correct items were more effective than those applied to incorrect items, and (b) vowels were more effective than consonants. The first asymmetry is consistent with earlier studies (Kausler et al., 1970; Kausler et al., 1975) showing significant facilitation when a rule applied to the right items only but not when it applied to the wrong items only. Kausler et al. (1975) have suggested that right items are processed at a deeper level and that, accordingly, shared features are more likely to be extracted. The present vowel-consonant asymmetry is not readily interpretable. It cannot be attributed simply to a bias to select words beginning with vowels, as each rule subgroup was compared with the appropriate control subset. Rather, the initial-vowel rule was more effective than the initial-consonant rule. Among the factors that might have contributed to this outcome are the following: intraset similarity, -either phonetic or typographical, may, be higher among the.vowels than among the consonants; initial-vowel words may be more distinctive, as English words are less likely to start with vowels than with consonants: and four vowels are a proportionately larger sample of the entire vowel population than are four consonants of the entire consonant population.

As shown by the analysis excluding the four aware subjects. awareness-defined in terms of verbalization of the rule in the final inquiry-was not a necessary condition for effective operation of the initial-vowel rule in VD learning. A similar result was obtained with a relational rule based on connotative meaning (Schwartz, 1974). In addition, Kausler and Farzanegan (1969), using pre-experimental word frequency as the basis for correctness. reported that few subjects seemed aware of any rule. It appears that a rule can facilitate VD learning in subjects who manifest no awareness of that rule or of any correlated rule which might have facilitated performance. Presumably this state of affairs would hold only when the rule is based on a common feature which is of intermediate salience. If the common feature were very obvious, subjects would become aware of it at once; if very obscure, it is unlikely that learning would be helped thereby.

\section{REFERENCES}

Deichmann, J. W., Minnigerode, F. A., \& Kausler, D. H. Selection strategies and reversal-nonreversal shifts in verbal discrimination transfer. Psychonomic Science, 1970, 18, 209-210.

Kausler, D. H., Erber, J. T., \& Olson, G. A. Taxonomic instances as right or wrong items and selection strategies in verbal discrimination learning. American Journal of Psychology, $1970,83,428-435$.

Kausler, D. H., \& Farzanegan, F. Word frequency and selection strategies in verbal-discrimination learning. Journal of Verbal Learning and Verbal Behavior, 1969, 8, 196-201.

Kausler, D. H., Majcher, L. L., \& Conover, J. N. Schematic exemplars as items in multiple-item recognition learning. Bulletin of the Psychonomic Society, 1975, 6, 472-474.

SCHWARTz, M. Verbal discrimination as a concept-attainment task using the evaluative dimension. Journal of Experimental Psychology, 1974, 102, 415-422.

(Received for publication February 9, 1976.) 